\title{
ELECTROPHORETIC CHANGES IN THE SERUM PROTEIN PATTERNS OF PATIENTS WITH SCARLET FEVER AND RHEUMATIC FEVER ${ }^{1}$
}

\author{
By VINCENT P. DOLE,2 ROBERT F. WATSON,2 AND SIDNEY ROTHBARD, wITH \\ THE TECHNICAL ASSISTANCE OF ESTHER BRAUN AND KENNETH WINFIELD
}

(From the United States Navy Research Unit at the Hospital of The Rockefeller Institute for Medical Research)

(Received for publication January 30, 1945)

It is generally recognized that rheumatic -fever is usually, if not always, preceded by a group $\mathrm{A}$ streptococcal infection. On the other hand, only a few of those individuals who suffer streptococcal infections develop clinical attacks of rheumatic fever. This fact makes it probable that the development of rheumatic fever also involves some peculiarity in the host reaction to the infection. Although immunological changes have been shown to occur in the sera of patients with streptococcal infections and rheumatic fever (1 to 8$)$, there has been no systematic study of the alterations in the overall pattern of serum proteins under these conditions. Since the changes in the serum protein pattern furnish additional data for a description of the host reaction, it seemed desirable to follow these changes in individuals who developed rheumatic fever following scarlet fever and in a control group of patients who had scarlet fever but did not develop rheumatic fever.

\section{MATERIAL AND METHODS}

Sera were collected at weekly intervals throughout the hospital stay of 110 patients originally admitted with scarlet fever. These sera were stored in sterile tubes at $4^{\circ} \mathrm{C}$. without preservative. Also at weekly intervals, the white blood cell count, erythrocyte sedimentation rate, and serum antistreptolysin 0 titer were determined; an electrocardiogram was taken, and cultures were made from the nose and throat. Six subjects were selected for electrophoretic study. This group, limited in size by the time required for analyses, was chosen to provide clinically typical examples of rheumatic fever following scarlet fever with comparable controls. None of the patients had previously suffered rheumatic fever or chorea.

The electrophoretic analyses were made in diethylbarbituric acid buffer $(\mu=0.1, \mathrm{pH}=8.6)$ (9). Normal plasma standards, obtained by this method (10), were

1 The Bureau of Medicine and Surgery does not necessarily undertake to endorse the views or opinions which are expressed in this paper.

2 Lieutenant Commander, Medical Corps, United States Naval Reserve. adjusted to a serum basis for the present study on the assumption that the loss of fibrinogen removed 5 per cent of the total protein. As a result of storage of sera, some specimens were as old as $\mathbf{1 8}$ months when analyzed. It is assumed that aging of the sera did not cause electrophoretically detectable changes except a slight reduction of $\beta$ globulin due to dissociation of lipid. Probably for this reason the usual $\beta$ globulin disturbance in the descending channel was absent in aged sera, although present in fresh specimens. The use of aged sera for a quantitative study cannot be justified by direct evidence, but it appeared quite definite that observed changes correlated with the stage of the disease rather than merely with the age of the serum sample.

The erythrocyte sedimentation rate (ESR) was determined by the Westergren method (11), using 3.8 per cent sodium citrate as anticoagulant.

The serum antistreptolysin 0 titer (ASO) was measured by the dilution technique of Todd (12) as modified by Hodge and Swift (13).

Nose and throat cultures were made in duplicate on fresh rabbit and sheep blood agar plates. The hemolytic streptococci were grouped and typed by the precipitin technique (14).

Sera from 3 different patients were absorbed with 18hour cultures of streptococci in attempts to elucidate the nature of $\gamma$ globulin rise. The strains had been preserved in the dried state, according to the technique of Swift (15). In each absorption, a mixture of 2 parts of serum with 1 part of packed, living bacterial cells (washed once with sterile saline) was incubated 30 minutes at $37^{\circ} \mathrm{C}$. Electrophoretic analyses were then made on absorbed serum, cleared by centrifugation, and on an unabsorbed control portion.

\section{Subjects of the study}

Rheumatic Fever (Figure 1), J. K. (No. 11371), a 25-year-old male, was admitted on the 4th day of illness with moderately severe scarlet fever due to group A, type 19 hemolytic streptococcus. From the 7 th to 18 th day of his illness, he was given sulfadiazine in treatment of a marked purulent nasal discharge and continued low grade fever. While under this treatment he became asymptomatic and afebrile. On the 22 nd day of his illness, however, he developed anorexia, and general malaise, followed in several days by fever, and migratory joint pains with a secondary rise in the erythrocyte sedimentation rate and first degree $\mathrm{A}-\mathrm{V}$ heart block. These 


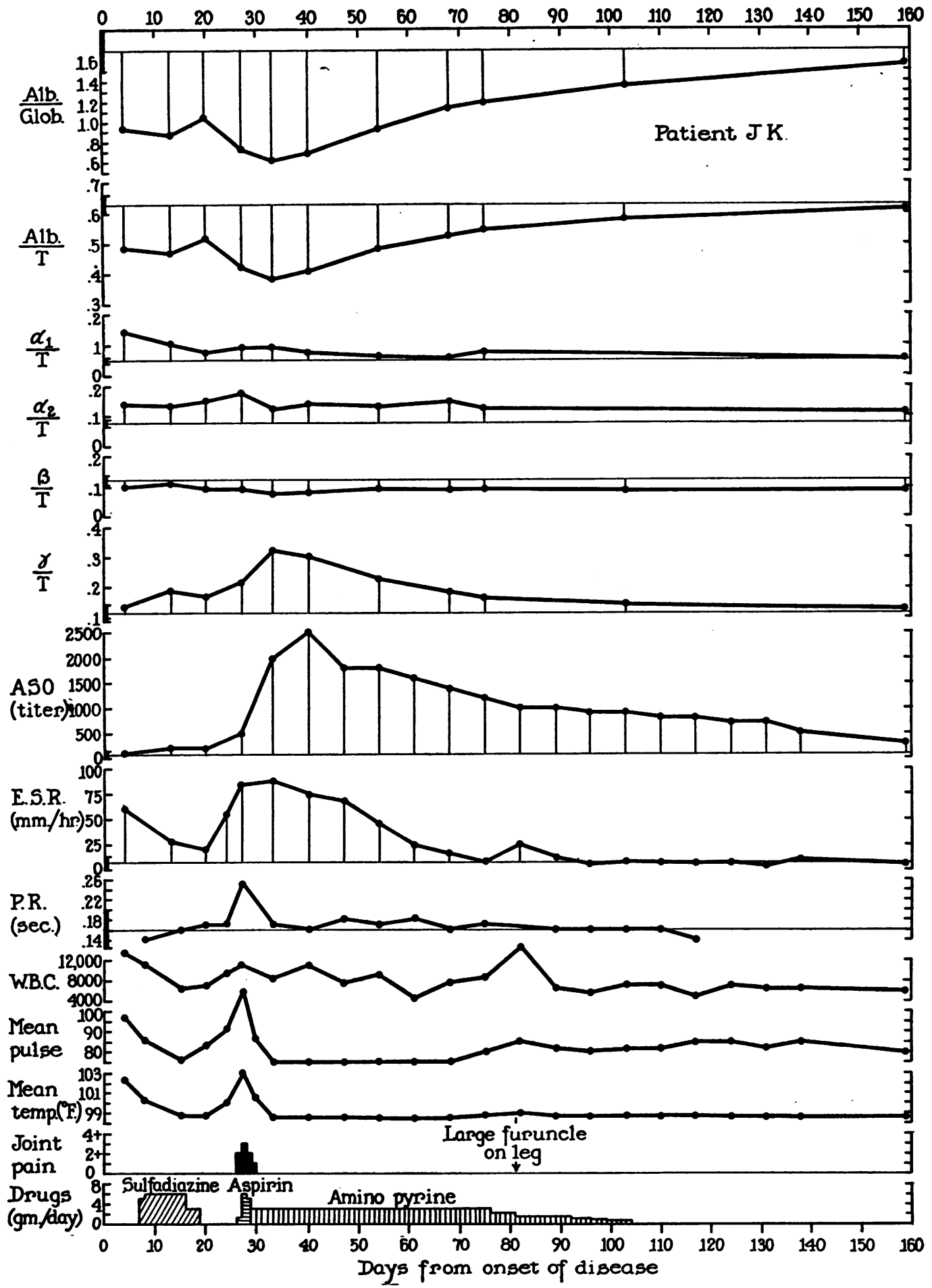

Fig. 1. Rheumatic Fever

From the top downwards, the ordinates indicate the albumin: globulin ratio $\left(\frac{\text { Alb. }}{\text { Glob. }}\right)$, the concentrations of serum protein components relative to the total protein $(T)$ as measured from the areas of the electrophoretic patterns, the titer of antistreptolysin 0 (ASO), the erythrocyte sedimentation rate (E.S.R.), the electrocardiographic P-R interval (P.R.), the white blood cell count (W.B.C.), and clinical data. Horizontal lines show normal average values, with normal ranges indicated by brackets at the left. 


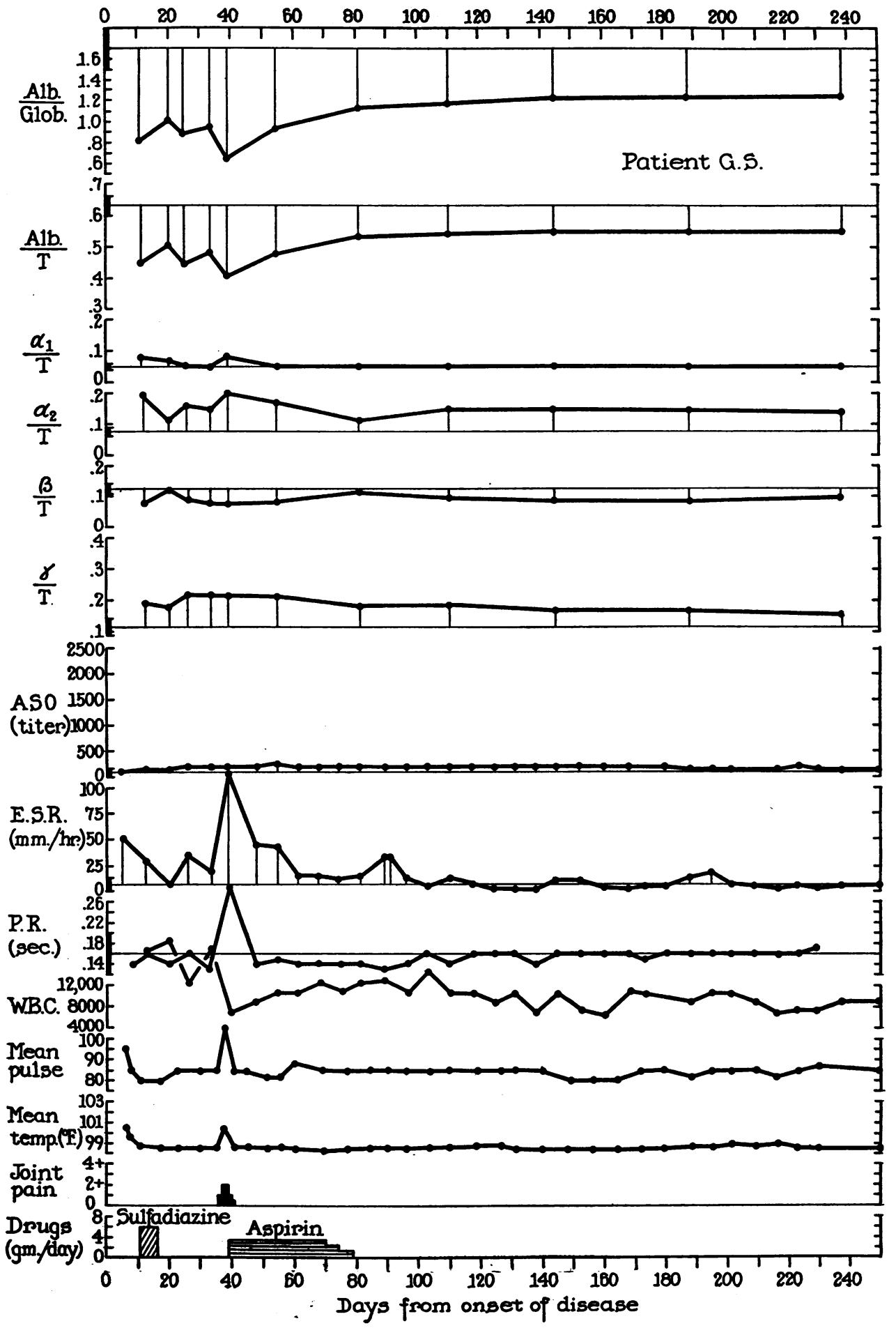

Fig. 2. Rheumatic Fever

Symbols as in Figure 1. 


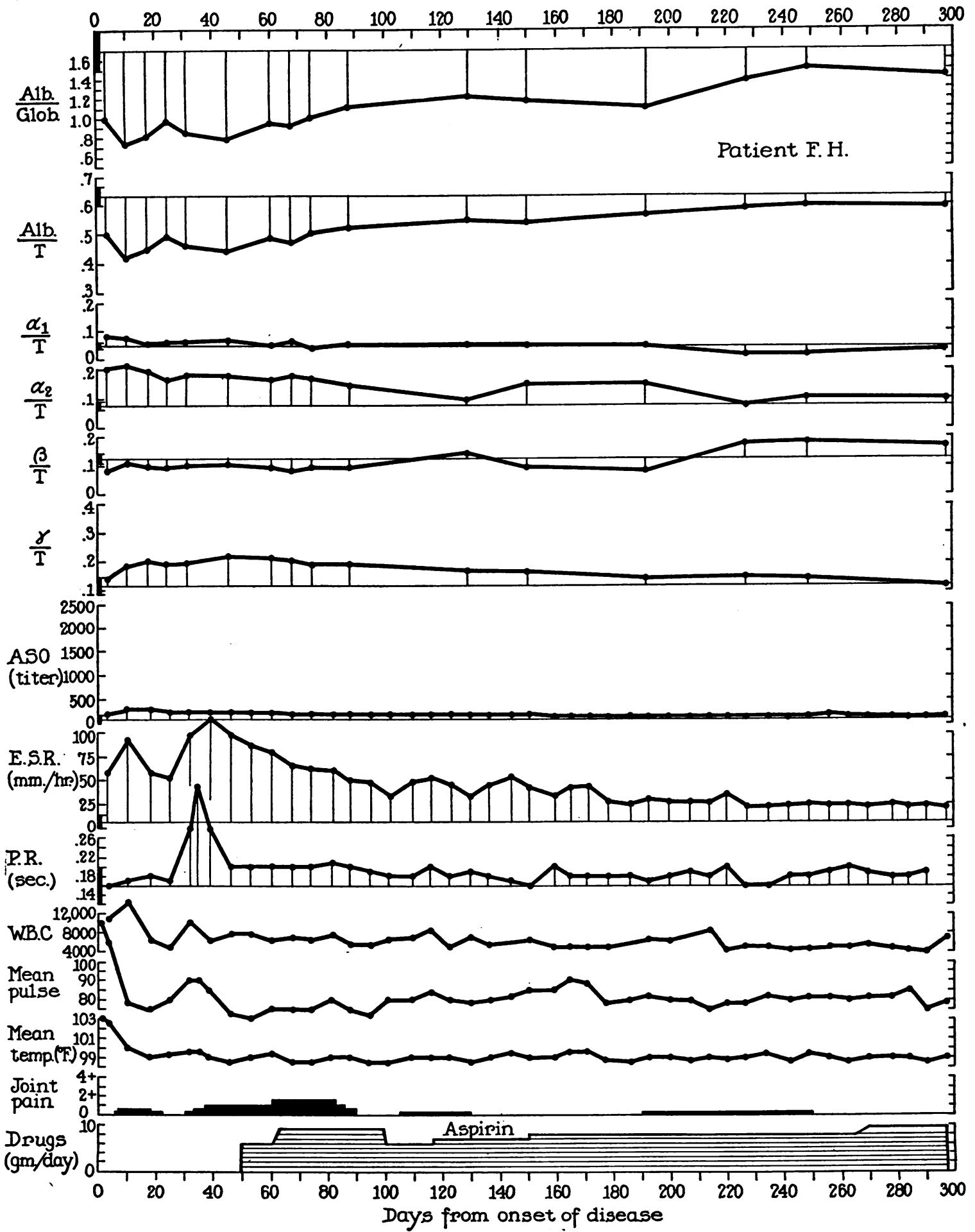

Fig. 3. Rheumatic Fever with a Protracted Course

Symbols as in Figure 1. 
symptoms abated under treatment with aspirin, for which aminopyrine was later substituted because of abdominal discomfort. Convalescence was thereafter uneventful except for a transient disturbance due to a furuncle on the leg. This patient was selected for study because he developed a typical attack of rheumatic fever with a single cycle of activity and a relatively high antistreptolysin 0 titer following scarlet fever.

Bacteriology. Nose and throat cultures, done weekly, showed the persistence of type 19 streptococci from admission to the 106th day. In addition, two other group A strains were recovered: a type 6 on the 7th day and another strain, not belonging to one of the known types, on the 19th day. The type 19 strain was presumed to have been the cause of the scarlet fever since the patient contracted his disease during an epidemic due to this type, which was also recovered in pure culture on admission. The unidentified type persisted throughout the hospital stay.

Rheumatic Fever (Figure 2), G. S. (No. 11328), a 32year-old male, was admitted on the 5 th day of a mild scarlet fever attack due to group A, type 19 hemolytic streptococcus. Within 3 days he became asymptomatic. From the 12th to 17 th day of illness he received sulfadiazine in an unsuccessful attempt to clear his carrier state. On the 20 th day he was asymptomatic with a normal erythrocyte sedimentation rate, but he had a leukocytosis of 20,000 , and showed in the electrocardiogram diphasic T-waves in leads II and III with low amplitude of the $\mathrm{T}$-waves in the limb leads. On the 35th day, he developed pains in the left shoulder and both wrists, with fever, increased pulse, partial A-V heart block, and a secondary rise in the erythrocyte sedimentation rate. Involvement of other joints occurred to the 38th day when he was started on salicylates with prompt clinical response. During the month following withdrawal of this medication on the 78th day, the erythrocyte sedimentation rate became elevated but he remained asymptomatic. Through the subsequent 4 months he remained asymptomatic with a normal sedimentation rate, but continued to have slight leukocytosis, and changes in the form and direction of the T-waves in leads I and II of the electrocardiogram. During the final month, these abnormalities were absent. At no time was there evidence of valvular disease. This patient was selected for study as a typical rheumatic fever subject. In contrast to the history of patient J. K., the onset of his rheumatic fever was more insidious, the course was more protracted, and the rise of antistreptolysin 0 titer was only slight.

Bacteriology. The type 19 streptococcus was found in weekly nasopharyngeal cultures throughout his entire hospital stay.

Rheumatic Fever (Figure 3), F. H. (No. 11574), a 23year-old female, was admitted on the 2nd day of her illness with severe scarlet fever due to group A, type 17 hemolytic streptococcus. During the next 4 days she continued to appear toxic, then gradually improved but did not become afebrile until after the 15 th day of illness. The appearance of pain in the cervical spine on the 5 th day of illness was followed by variable, low-grade pains in the knees, left wrist, and left shoulder, during the ensuing 3 weeks. A typical attack of rheumatic fever developed about the 30th day with fever, increased pulse rate, leukocytosis, partial $\mathrm{A}-\mathrm{V}$ heart block, secondary rise in the erythrocyte sedimentation rate, and recurrent joint symptoms. At this time, there was a systolic murmur at the apex. On the 50th day, she was started on aspirin therapy, which was continued over the following 8 months. Despite the medication she continued to have intermittent joint pains until the last month. During the 4th month, an aortic diastolic blow was first noted; at this time a subcutaneous nodule was found over the right patella. At the time of discharge to another hospital on the 299th day, she was asymptomatic on 9.5 grams of aspirin daily. The heart was of normal size with an aortic diastolic blow. She was selected for study as an example of rheumatic fever with a protracted course.

Bacteriology. The type 17 streptococcus was found in nasopharyngeal cultures throughout the entire hospital stay.

Uncomplicated Scarlet Fever (Figure 4a), W. K. (No. 11341), a 19-year-old male, was admitted on the 3rd day of illness with fairly severe scarlet fever due to group A, type 19 hemolytic streptococcus. He became asymptomatic by the 6th day of illness, but was started on sulfadiazine at this time in an unsuccessful attempt to clear his carrier state. After an uneventful convalescence, he was discharged on the 48th day. This patient represents a typical severe scarlet fever patient with an uneventful convalescence.

Bacteriology. The original type of streptococcus was found in weekly cultures of the nose and throat throughout the period of observation.

Uncomplicated Scarlet Fever (Figure 4b), J. J. (No. 11291), a 20-year-old male, was admitted on the 3rd day of illness with severe scarlet fever due to group A, type 19 hemolytic streptococcus. A few days after admission he became asymptomatic but was started on sulfadiazine, followed by nose and throat sprays of Zephiran and tyrothricin in unsuccessful attempts to clear his streptococcal carrier state. After an uneventful convalescence, he was discharged on the 77th day. This patient, like W. K., was representative of patients with uncomplicated scarlet fever.

Bacteriology. The original type of streptococcus was found in weekly cultures of the nose and throat throughout the period of hospitalization.

Scarlet Fever Complicated with Purulent Sinusitis (Figure 4c), D. S. (No. 11312), a 23-year-old male, was admitted on the 3rd day of illness with severe scarlet fever due to group A, type 19 hemolytic streptococcus. On symptomatic treatment only, he showed gradual improvement until the 13th day, when he first complained of pain over the right side of the face. Examination revealed tenderness and edema over the right antrum; $x-$ rays on the following day showed clouding of the right maxillary and ethmoid sinuses. Within 48 hours after the institution of sulfadiazine therapy and saline irrigations of the right antrum, he became afebrile and asymptomatic. He remained so thereafter until discharge on 


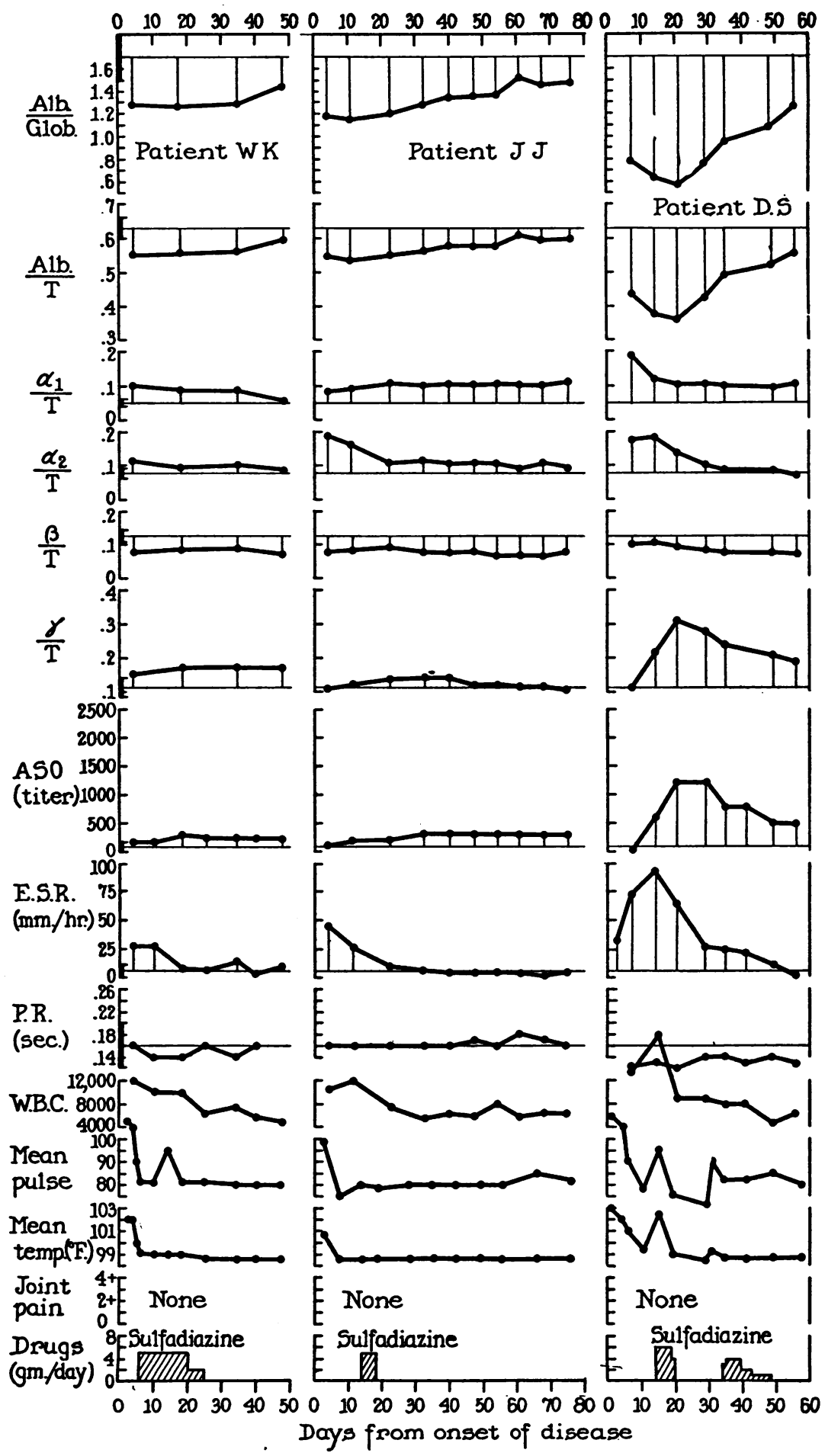

Fig. 4. Scarlet Fever without Rheumatic Fever

Patients W. K. and J. J. were uncomplicated; patient D. S. had purulent sinusitis as a complication. Symbols as in Figure 1. 
the 59th day. The patient was selected for study to illustrate the effect of a purulent complication of scarlet fever in a non-rheumatic subject.

Bacteriology. The original type of streptococcus was found in weekly nose and throat cultures to the 34th day and in the purulent sinus exudate on the 15th day. No hemolytic streptococci were found in nose and throat cultures after the 34 th day.

\section{OBSERVATIONS AND DISCUSSION}

The electrophoretic changes in the serum protein patterns of 2 patients with uncomplicated scarlet fever, one patient with a purulent complication, and 3 who developed typical attacks of rheumatic fever, are illustrated in Figures 1 through 4. Marked, systematic changes from the normal serum protein pattern (10) were found in all cases. The relative concentration of albumin was depressed by the attack of scarlet fever, with a consequent marked reduction in the albuminglobulin ratio. The slow rise toward normal in convalescence from scarlet fever was apparently delayed by the development of clinical rheumatic fever. Of the globulins, $a_{1}$ was elevated with the scarlet fever attack to a relative concentration 2 to 3 times the normal value, and fell toward normal as rapidly in rheumatics as in non-rheumatics. The $a_{2}$ globulin, likewise elevated with the scarlet fever, tended to maintain an abnormal elevation in the rheumatic patients, in accord with the finding of another group of workers (16). The $\beta$ globulin showed no characteristic change.

Of particular interest are the changes in the relative concentration of $\gamma$ globulin since some antibodies, at least, are contained in this fraction of the serum (17). Indirect support of the pre- sumption that specific antibodies induced by the streptococcal infection also move with the $\gamma$ globulin is found in the general correlation between the titer of antistreptolysin 0 and the relative concentration of $\gamma$ globulin (Figures 1 to 4 ). It should not be assumed, however, that the rise of $\gamma$ globulin was quantitatively due to increase of this antibody alone, since in all probability there were increased amounts of other antibodies, both specific for the infection and anamnestic. An estimation of the amount of antistreptolysin 0 present could not be made for want of a suitable technique for absorption with the streptolysin 0 .

Absorption experiments were done on the sera of 3 patients to determine whether or not an appreciable part of the $\gamma$ globulin rise might be due to antibodies directed toward the somatic antigens of the streptococcal cell. In each of the 3 cases, the serum was absorbed with the living streptococcal cells of the strain that had induced the patient's attack of scarlet fever. There was sufficient serum from only 1 of the patients to include a heterologous-type control. As may be seen in Table I, no significant changes were found in the relative concentrations of $\gamma$ globulin or of the other protein components following these absorptions. A decrease of at least 10 per cent in the concentration of $\gamma$ globulin would have had to occur to be significant. The slight decreases in the titer of. antistreptolysin 0 are about at the limit of experimental error. If not fortuitous, they are probably due to small amounts of streptolysin introduced with the living streptococci.

Whatever may be the explanation of the rises in $\gamma$ globulin, it is clear that these increases in

TABLE I

Absorptions of sera with streptococci

The first 5 rows indicate the fraction of the total serum protein $(T)$ furnished by the serum protein components, as estimated from the areas of the electrophoretic patterns. ASO is antistreptolysin O titer

\begin{tabular}{l|c|c|c|c|c|c|c}
\hline \hline & \multicolumn{3}{|c|}{ Patient A. W.t } & \multicolumn{2}{c|}{ Patient D. S. } & \multicolumn{2}{c}{ Patient J. K. } \\
\cline { 2 - 6 } & $\begin{array}{c}\text { Unabsorbed } \\
\text { control }\end{array}$ & $\begin{array}{c}\text { Absorbed with } \\
\text { patient's strain }\end{array}$ & $\begin{array}{c}\text { Absorbed with } \\
\text { heterologous type }\end{array}$ & $\begin{array}{c}\text { Unaborbed } \\
\text { control }\end{array}$ & $\begin{array}{c}\text { Absorbed with } \\
\text { patient's strain }\end{array}$ & $\begin{array}{c}\text { Unabsorbed } \\
\text { control }\end{array}$ & $\begin{array}{c}\text { Absorbed with } \\
\text { patient's strain }\end{array}$ \\
\hline Alb./T & 0.436 & 0.432 & 0.402 & 0.448 & 0.447 & 0.482 & 0.508 \\
$\alpha_{1} / T$ & 0.091 & 0.108 & 0.105 & 0.102 & 0.095 & 0.046 & 0.087 \\
$\alpha_{2} / T$ & 0.193 & 0.193 & 0.214 & 0.106 & 0.102 & 0.155 & 0.094 \\
$\beta / T$ & 0.090 & 0.099 & 0.095 & 0.089 & 0.093 & 0.094 & 0.075 \\
$\gamma / T$ & 0.190 & 0.178 & 0.184 & 0.264 & 0.262 & 0.232 & 0.237 \\
ASO & 600 & 500 & 500 & 1000 & 800 & & \\
\hline
\end{tabular}

$\dagger$ This patient, not otherwise included in the study, had a typical acute attack of rheumatic fever following a group A, type 5 streptococcal pharyngitis. 
relative concentration were not correlated with rheumatic fever as such, since comparable increases in this fraction were observed in the nonrheumatic who had a purulent sinusitis (D. S.), and in a rheumatic (J. K.), while slight to moderate increases only were found in 2 non-rheumatics (W. K., J. J.) and in 2 rheumatics (G. S., F. H.).

The mobilities of the globulin components, relative to the albumin mobility, are given in Table II. These values are not precise, owing in part

TABLE II

The mean relative mobilities of the globulin components relative to albumin

(Normal standards are calculated from data in reference 10)

\begin{tabular}{l|c|c|c|c}
\hline \hline & $\alpha_{\mathbf{1}}$ & $\alpha_{\mathbf{2}}$ & $\beta$ & $\gamma$ \\
\hline Normal & 0.854 & 0.687 & 0.476 & 0.172 \\
Rheumatic & 0.861 & 0.674 & 0.509 & 0.197 \\
Scarlet fever, & 0.851 & 0.679 & 0.517 & 0.213 \\
non-rheumatic & & & & \\
\hline
\end{tabular}

to the variations in protein concentration in the samples analyzed. It is also possible that variations in the relative mobilities resulted from aging of the sera, although no correlation could be found between change in relative mobilities and age of sera at the time of analysis. It appears, despite these uncertainties, that there were no significant differences in relative mobilities to distinguish the sera of the 3 rheumatic subjects from those of the 3 non-rheumatic scarlet fever controls.

The serum protein changes in the non-rheumatic patients presumably reflected a host reaction to infection with hemolytic streptococci. In the rheumatic subjects, although the original changes can be explained equally well by the same host reaction, the reason for the great delay in return to normal is not evident. Persistence of the microorganism in the nasopharynges was found in the non-rheumatic, as well as in the rheumatic subjects. In view of the non-specific nature of the observed serum protein changes, it can only be said that the protracted changes reflected the physiological disturbances associated with continued rheumatic inflammation. Except, therefore, for the greater duration of abnormalities in these patients, there appeared to be no electrophoretic changes that distinguished the sera of patients who developed rheumatic fever after scarlet fever from the sera of patients who had scarlet fever alone.

\section{SUMMARY AND CONCLUSIONS}

1. The changes in the serum protein pattern were followed by electrophoretic analyses in 6 patients originally admitted with scarlet fever, 3 of whom subsequently developed rheumatic fever.

2. Systematic, marked changes of a qualitatively similar nature were found in both rheumatic and non-rheumatic subjects; an early depression of albumin with rises in the $a_{1}$ and $a_{2}$ globulins, and a delayed rise in $\gamma$ globulin. These abnormalities were, however, more prolonged in the rheumatic patients.

3. A general correlation was found between the increase in $\gamma$ globulin and the titer of antistreptolysin 0 . In 3 cases tested, no detectable amount of $\gamma$ globulin could be removed by absorption with the living streptococcal cells of the strain that had induced the patient's attack of scarlet fever.

4. No significant changes were found in the relative mobilities of the globulin components either in the rheumatic or non-rheumatic group.

5. It was concluded that there were no changes in the serum protein pattern, detectable by the method used, to distinguish rheumatic from nonrheumatic patients. The greater duration of abnormalities in the rheumatics was assumed to be a reflection of the physiological disturbances associated with continued rheumatic inflammation.

The authors are indebted to Drs. T. Shedlovsky and D. A. MacInnes for their generosity in making available the electrophoretic equipment used in this study.

\section{BIBLIOGRAPHY}

1. Todd, E. W., Antihaemolysin titres in haemolytic streptococcal infections and their significance in rheumatic fever. Brit. J. Exper. Path., 1932, 13, 248.

2. Tillett, W. S., The occurrence of antifibrinolytic properties in the blood of patients with acute hemolytic streptococcus infections. J. Clin. Invest., 1935, 14, 276.

3. Hadfield, G., Magee, V., and Perry, C. B., The lysis of fibrin by Streptococci: its application to the problems of rheumatic infection in children. Lancet, 1934, 1, 834.

4. Swift, H. F., and Hodge, B. E., Type-specific anti-M precipitins in rheumatic and non-rheumatic patients with hemolytic streptococcal infections. Proc. Soc. Exper. Biol. and Med., 1936, 34, 849.

5. Swift, H. F., Rheumatic heart disease. Pathogenesis and etiology in their relation to therapy and prophylaxis. Medicine, 1940, 19, 417. 
6. Coburn, A. F., Observations on the mechanism of rheumatic fever. Lancet, 1936, 2, 1025.

7. Coburn, A. F., and Pauli, R. H., A precipitinogen in the serum prior to the onset of acute rheumatism. J. Exper. Med., 1939, 69, 143.

8. Kuttner, A. G., and Lenert, T. F., The occurrence of bacteriostatic properties in blood of patients after recovery from streptococcal pharyngitis. J. Clin. Invest., 1944, 23, 151.

9. Longsworth, L. G., Recent advances in the study of proteins by electrophoresis. Chem. Rev., 1942, 30, 323.

10. Dole, V. P., The electrophoretic patterns of normal plasma. J. Clin. Invest., 1944, 23, 708.

11. Westergren, A., Studies on the suspension stability of the blood in pulmonary tuberculosis. Acta med. Scandinav., 1921, 54, 247.

12. Todd, E. W., Antigenic streptococcal hemolysin. J. Exper. Med., 1932, 55, 267.
13. Hodge, B. E., and Swift, H. F., Varying hemolytic and constant combining capacity of streptolysins; influence on testing for antistreptolysin. J. Exper. Med., 1933, 58, 277.

14. Swift, H. F., Wilson, A. T., and Lancefield, R. C., Typing group A hemolytic streptococci by $M$ precipitin reactions in capillary pipettes. J. Exper. Med., 1943, 78, 127.

15. Swift, H. F., A simple method for preserving bacterial cultures by freezing and drying. J. Bact., 1937, 33, 411.

16. Longsworth, L. G., Shedlovsky, T., and MacInnes, D. A., Electrophoretic patterns of normal and pathological human blood serum and plasma. J. Exper. Med., 1939, 70, 399.

17. Enders, J. F., The concentrations of certain antibodies in globulin fractions derived from human blood plasma. J. Clin. Invest., 1944, 23, 510. 